\title{
MULHERES EM CIÊNCIA E TECNOLOGIA: ASCENSÃO LIMITADA
}

\section{Thereza Amélia Soares}

Departamento de Química Fundamental, Universidade Federal de Pernambuco, 50670-901, Cidade Universitária, Recife - PE

Recebido em 4/10/99; aceito em 22/11/00

\begin{abstract}
WOMEN IN SCIENCE AND TECHNOLOGY: RESTRICTED SUCCESS. In contemporary times, women can choose freely to enter any professional field. Along the way they come across stumbling blocks that make their progress difficult. Most of these difficulties are not gender-specific, yet women encounter them more consistently than do men. It is remarkably true for the areas of Science and Technology. However, it is not straightforward to evaluate and to obtain an accurate measure of the effects of gender bias. The factors and consequences associated with the phenomena are multiple, with many shades of regionalism as we look at different countries. Despite of the absence of detailed studies regarding the situation in Brazil, it seems unlikely we would be an exception to a world pattern. In this article, some causes as well as current actions around the world to fight gender bias are presented.
\end{abstract}

Keywords: women in science; gender bias; strategies.

\section{INTRODUÇÃO}

A representação desproporcional de mulheres em C\&T não é um tema novo em ciência e tem sido amplamente abordado na última década ${ }^{1,2}$. As causas para o problema são complexas e com múltiplas facetas, sejam estas de ordem sócio-cultural, econômica ou cognitiva ${ }^{3}$. No entanto, através de medidas que estimulam o aprendizado de matemática e áreas correlatas em um período precoce do desenvolvimento feminino, assim como políticas de incentivo a jovem cientistas, esta situação tem sido gradativamente revertida ${ }^{3,4}$. Em 1995 , a proporção de mulheres obtendo diplomas em áreas de C\&T em instituições americanas atingiu o valor de $46 \%{ }^{4}$. Certamente, este valor é uma superestimativa visto que a classificação como ciências pelo National Science Foundation inclui as áreas de psicologia e ciências sociais, onde tradicionalmente a proporção de mulheres sobrepõem-se à proporção de homens ${ }^{4}$. Ainda assim, em 1995 um terço do total de diplomas em física concedidos por instituições americanas foram obtidos por mulheres ${ }^{4}$. Se um número significativo de mulheres são bem sucedidas na obtenção de diplomas em áreas de C\&T, porque tão poucas mulheres são encontradas em posições acadêmicas permanentes?

Recentemente, o Conselho de Pesquisa Médica da Suécia financiou um amplo estudo na tentativa de identificar os fatores determinantes deste problema ${ }^{5}$. A publicação dos resultados em 1997 surpreendeu a comunidade científica ao revelar uma forma sutil de discriminação sexual através do mecanismo pelo qual financiamentos de projetos e laboratórios de pesquisas são concedidos. Entre outras estatísticas, o relatório mostrou que para obter suporte financeiro, pesquisadoras devem ser em média 2,2 vezes mais produtivas do que seus colegas do sexo masculino ${ }^{5}$. Como consequência, uma série de estudos similares em países europeus como Reino Unido, Finlândia e Dinamarca foram realizados indicando que o problema é bastante generalizado ${ }^{6,7}$. Mais recentemente, os resultados de uma pesquisa iniciada há 5 anos por um grupo de 15 professoras no Massachussets Institute of Technology (MIT) foram divulgados corroborando as conclusões do grupo sue$\operatorname{co}^{8,9}$. Com a publicação destes dados estatísticos, obtidos por pesquisadores e instituições respeitadas no meio científico,

Present adress: Pacific Northwest National Laboratory, 906 Battelle Boulevard Math MS-83 K1, 99352 Richland, WA, USA. parece difícil justificar a ausência de mulheres em ciência devido à inerente inaptidão feminina para ciências exatas. Contrariamente, as causas parecem resultar da inadequação das estruturas institucionais às quais estas cientistas pertencem ${ }^{9-12}$.

A grande maioria das publicações relacionadas à representação feminina em áreas de C\&T referem-se a estudos realizados em países europeus e norte-americanos ${ }^{1,2,3,13}$ e por esta razão os dados estatísticos apresentados neste artigo são representativos de tais países. No Brasil é difícil avaliar a situação devido ao número restrito de publicações na área, a maioria destes publicados anteriormente aos anos 90. Um detalhado estudo publicado por Azevedo et $a l .{ }^{45}$, baseado em dados do CNPq e FINEP mostrou que em 1988 a mulher representava um terço da força produtora de conhecimento científico no país. Neste mesmo período, a porcentagem de mulheres em C\&T variou em torno de $28 \%$ excluindo a área de ciências biológicas onde mulheres constituíam $48 \%$ dos recursos humanos em ciência e ensino no país ${ }^{45}$. No entanto, apenas $8 \%$ e $23 \%$ dos consultores científicos da FINEP nas áreas de ciências exatas e ciências biológicas pertenciam ao sexo feminino ${ }^{45}$. Nas engenharias nenhum dos consultores eram do sexo feminino. Atualmente, apenas $29 \%$ dos pesquisadores com bolsa de produtividade do $\mathrm{CNPq}$ na área de química são do sexo feminino, enquanto que aproximadamente $53 \%$ das bolsas de doutorado em química no país pela mesma instituíção pertencem ao sexo feminino ${ }^{46}$. Nenhum dos membros do Comitê de Assessoramento de Química do CNPq no presente momento é do sexo feminino ${ }^{46}$.

Apesar do número restrito e pouco acessível de estudos sobre a atuação feminina em áreas de $C \& T$, é razoável supor, com base na observação do número de mulheres ocupando posições permanentes em departamentos de engenharia, matemática, física e química brasileiros, que o Brasil não constitui uma exceção à tendência constatada em outros países. Seria surpreendente a descoberta que o Brasil, um país onde fenômenos sociais como o machismo e o marianismo (vide glossário) estão profundamente enraigados culturalmente, constitui uma exceção à regra ${ }^{13}$. Desta forma, a proposta deste artigo é meramente estimular a discussão e pesquisas mais aprofundadas sobre a presença feminina em C\&T.

\section{ORIGENS PARA O PROBLEMA: ASPECTOS SÓCIO- CULTURAIS VERSUS DIFERENÇAS BIOLÓGICAS}

Duas perspectivas diferentes têm sido propostas para explicar as causas da representação desproporcional de mulheres em C\&T. Uma delas atribue o problema a diferenças biológi- 
cas, cognitivas ou de socialização entre os dois sexos ${ }^{14,15}$. Os argumentos mais comuns em favor desta hipótese são que mulheres não possuem controle emocional para suportar as pressões frequentes em cargos de comando, que mulheres não tomam decisões objetivas e são socialmente educadas para serem "protegidas" e desta forma não adquirem a "agressividade" necessária para competir. Uma segunda perspectiva propõe que os padrões institucionais determinam as escolhas individuais, que por sua vez mantem e reforçam ciclicamente estes mesmos padrões ${ }^{14,15}$. Em outras palavras, a estrutura das organizações não propicia o sucesso profissional do sexo feminino. Sob este ponto-de-vista, a questão de mulheres em C\&T é o resultado de estruturas institucionais inapropriadas e não da inaptidão feminina para as áreas de $C \& \mathrm{~T}^{13}$.

Durante os primeiros anos de escola até o início do ginásio, o interesse ou habilidade para matemática não variam significativamente em função do sexo ${ }^{16,17}$. Contudo, ao final dos anos de ginásio, uma diferença crescente na capacidade de aprendizagem de matemática bem como ciências em geral é evidente entre os dois sexos ${ }^{18-21}$. Indivíduos do sexo masculino apresentam um melhor desempenho em matemática e áreas afins quando comparados ao sexo feminino. No entanto, estudos realizados em escolas norte-americanas mostram que estudantes de segundo grau com uma mesma história de disciplinas cursadas durante o período, não apresentam diferenças na aptidão para matemática ou ciências relacionadas à diferença de sexo ${ }^{22}$. Uma melhor compreensão destes fatos aparentemente contraditórios é dada pelo número de disciplinas em matemática cursadas durante os anos de colegial. Este é um critério aplicado em escolas norteamericanas para estimar a permanência de indivíduos em áreas de C\&T após o ingresso em cursos universitários ${ }^{23}$. Ao concluir o período correspondente ao colegial, indivíduos do sexo masculino cursaram substancialmente mais disciplinas em matemática e ciência que indivíduos do sexo feminino ${ }^{23}$. Com um preparo mais aprofundado em matemática e ciências, estudantes do sexo masculino estarão consequentemente mais aptos, e mais motivados para o ingresso em cursos universitários de C\&T.

Leslie et $a l .^{22}$ propõem que a representação desproporcional de mulheres em C\&T resulta principalmente do desinteresse em ciências e matemática durante a adolescência quando as bases do conhecimento formal nestas áreas são fundamentadas e não, da inaptidão feminina para ciências exatas. Fatores diversos e complexos são determinantes para este desinteresse ${ }^{22}$. Adolescentes do sexo feminino apresentam uma menor expectativa de sucesso profissional em áreas de C\&T, e posteriormente como profissionais na área são menos autoconfiantes que as demais profissionais do sexo feminino em outras atividades ${ }^{22,24}$. É interessante notar que adolescentes, especialmente do sexo masculino, mas também do sexo feminino, consideram que homens possuem uma capacidade superior de aprendizagem e percepção de ciências e matemática comparada às mulheres ${ }^{17,18,19,25}$. Em outras palavras, garotos superestimam a própria habilidade enquanto garotas subestimam sua habilidade. Até as primeiras décadas do século passado, um mesmo padrão comportamental foi observado na área esportiva, tradicionalmente considerada como domínio masculino, e onde mulheres estiveram presentes em número reduzido. Obviamente, esta situação não é mais verdadeira na presente época. Contudo, matemática e ciências continuam sendo considerados como domínios masculino ${ }^{21,26-28}$.

Certamente, diferenças biológicas existem e, ainda que os resultados na área sejam inconclusivos e não exista um consenso sobre a extensão destas diferenças que determinam a capacidade de aprendizagem de ambos os sexos, este é um fato que não pode ser ignorado ${ }^{3,29}$. No entanto, aspectos sociais previamente discutidos são fatores determinantes na representação desproporcional de mulheres em áreas de C\&T. Alguns destes aspectos são comuns à maioria dos países e parecem estar relacionados às dificuldades encontradas pela mulher em qualquer profissão bem como às dificuldades peculiares da carreira científica. Entre eles podemos enumerar: i) a dificuldade em conciliar as demandas da própria profissão e aquelas da profissão do parceiro. Mulheres aceitam mais facilmente uma mudança geográfica relacionada à profissão do parceiro do que os homens em relação à parceira. Este padrão é comum mesmo para casais nos quais ambos indivíduos são cientistas (o chamado $t$ wo-body problem $)^{30}$; ii) a sobrecarga devido ao acúmulo das tradicionais funções do lar e da profissão acadêmica. A solução encontrada por algumas universidades é a criação de creches no próprio local de trabalho e a implementação de práticas que permitam uma maior flexibilidade no horário de trabalho ${ }^{11,12}$; iii) o reduzido número de mulheres em cargos de decisão. Isto dificulta a implementação de políticas e medidas que estimulem uma maior participação feminina em ciência e tecnologia. Particularmente nos Estados Unidos e Europa, existem discrepâncias com respeito a salário, aposentadoria, espaço físico e condições de pesquisa em laboratórios entre cientistas do sexo feminino e masculino ${ }^{8,9,31,32}$. Certamente estas discrepâncias se refletirão na qualidade da pesquisa desenvolvida pelos dois sexos. Entretanto, este não parece ser um problema no Brasil; iv) o escasso reconhecimento dentro da própria comunidade científica. Marie Curie é um clássico exemplo a ser citado. Foi necessário a recusa de Pierre Curie em receber o prêmio Nobel sozinho para que a comissão reconhecesse a contribuição de Marie na área de radioatividade. Mais tarde, a contribuição essencial de Rosalind Franklin para a elucidação da estrutura do DNA foi completamente ignorada pela comissão do prêmio Nobel. Outros exemplos podem ser facilmente encontrados em qualquer livro bibliográfico sobre mulheres cientistas ${ }^{33}$.

Neste ponto, é interessante ressaltar que professoras universitárias mais jovens sentem-se mais integradas e apoiadas nos respectivos departamentos 8,9 . Para este grupo, o problema mais comum consiste em conciliar família e trabalho. Contudo, à medida que progridem nas respectivas carreiras acadêmicas, muitas destas cientistas sentem-se marginalizadas e excluídas de posições de efetiva capacidade de decisão ${ }^{8,9}$. A descoberta que emerge destes fatos é que, em cada geração, jovens pesquisadoras acreditam que a discriminação sexual é uma questão resolvida pelas gerações anteriores e que não as afetará.

\section{UM PROBLEMA SEM FRONTEIRAS GEOGRÁFICAS}

Como o debate promovido pela revista Nature mostrou recentemente, a baixa percentagem de mulheres em posições acadêmicas continua sendo um problema mundial ${ }^{3}$. O número de estudantes de ambos os sexos em cursos universitários de ciências biológicas no Reino Unido é aproximadamente o mesmo. Contudo, esta proporção está longe do equilíbrio nos cursos de física, matemática e ciências computacionais ${ }^{34}$. Do mesmo modo, um declínio persistente da percentagem de mulheres é observado ao longo das diferentes etapas da pós-graduação. Apenas 3$4 \%$ do corpo docente universitário em qualquer área de ciência e tecnologia no Reino Unido é constituído por mulheres ${ }^{35}$. O mesmo é válido para os Conselhos de Pesquisa e demais orgãos governamentais. O número de bolsas concedidas para mulheres pela Royal Society, Royal Society of Engineering ou ainda pelo Institute of Biology é menor que $10 \%{ }^{36}$. Mesmo em países do sul da Europa, como Portugal, Itália e Espanha, onde um número maior de mulheres ocupam posições permanentes em arquitetura, engenharia, matemática e ciências computacionais, a representatividade feminina é menor que $10 \%$.

A situação não é diferente na Alemanha ${ }^{35}$. Um total de $6,8 \%$ dos estudantes que obtiveram um diploma em ciências biológicas entre os anos de 1977 e 1979 conseguiram também obter a chamada habilitação ${ }^{11}$. Este exame é obrigatório para os estudantes que aspiram ensinar em Universidades ou Instituições de Pesquisa Alemãs. Naquele mesmo período, a proporção de estudantes do sexo feminino que obtiveram a habilitação foi de apenas $0,8 \%^{11}$. Como consequência, atualmente existem 
poucas mulheres ocupando posições de professor ou diretor de instituições de pesquisa. A maior parte destas pesquisadoras ocupam a mesma posição há 20 anos $^{11}$. Um outro fator agravante é a abundância de contratos de curta duração (3-5 anos) em contraposição ao reduzido número de posições permanentes. Ao término destes contratos, o pesquisador deve procurar uma outra instituição, a menos que tenha alcançado uma posição de Professor ou Diretor de Instituto.

Nos Estados Unidos, o número de mulheres com diploma de bacharelado em áreas de ciência e tecnologia apresenta um lento mas estável aumento, saindo de 38-39\% em meados de 1980 para $46 \%$ no ano de $1995^{4}$. Contudo, os índices variam dentro das diversas áreas de C\&T. Em 1995, mulheres obtiveram respectivamente $73 \%$ e $50 \%$ dos diplomas de bacharelado em psicologia e ciências biológicas/agriculturais, áreas onde tradicionalmente o sexo feminino é bem representado. Contudo, mulheres obtiveram apenas um terço dos diplomas de bacharelado em ciências físicas, ciências oceanográficas e ambientais, e em matemática e ciências computacionais ${ }^{4}$. Nas engenharias, este índice foi de apenas $17 \%$. Uma situação oposta é observada nas áreas não classificadas como C\&T, onde mulheres obtiveram mais da metade dos diplomas em $1996^{4}$.

Similarmente aos países europeus, também nos Estados Unidos é observado um decréscimo progressivo no número de mulheres a obter títulos de Mestre $(38 \%)$ e Doutor $(31 \%)^{4}$. Nas demais áreas, a percentagem de mulheres que obtêm diploma de bacharelado é similar à percentagem de mulheres que obtêm títulos de Mestre ou Doutor.

Dados estatísticos sobre a representação feminina em instituições acadêmicas na América Latina bem como no Brasil são escassos ${ }^{13,45}$. Os dados mais recentes referentes a América Latina foram publicados no relatório final da Conferência Mundial sobre Ciência organizada pela UNESCO em Bariloche, Argentina em $1998^{37}$.

Segundo o relatório da UNESCO, o número de mulheres ingressando em áreas de ciência e tecnologia nos países da América Latina tem aumentado na última década ${ }^{37}$. No entanto, persiste a concentração de profissionais do sexo feminino em áreas específicas como ciência sociais e psicologia, em detrimento das demais áreas ${ }^{37}$. Em países latinos, aspectos culturais consituem um obstáculo adicional. A associação do papel social da mulher aos tradicionais modelos e práticas típicas em sociedades patriarcais dificulta a qualificação profissional feminina, anteriormente mesmo ao ingresso em universidades. Em geral, as mulheres latino-americanas não consideram a estrutura de instituições acadêmicas discriminatórias, embora considerem-se segregadas a funções que são uma extensão do modelo feminino em sociedades patriarcais ${ }^{13}$. Um estudo realizado na Costa Rica indica que as mulheres em instituições acadêmicas daquele país apresentam uma visão mais positiva a respeito dos respectivos êxitos profissionais e status dentro de instituições quando comparadas às norte-americanas ${ }^{13}$. Fato este que parece estar relacionado ao referencial das mulheres americanas que, no caso, é o profissional do sexo masculino, enquanto que as costa-riquenhas consideram-se um subgrupo privilegiado dentro de uma sociedade machista ${ }^{13}$.

\section{POR QUE A PARTICIPAÇÃO FEMININA EM C\&T É IMPORTANTE?}

A maior participação feminina em C\&T pode implicar em transformações sociais e econômicas com um impacto favorável para a sociedade. O crescente interesse demonstrado pelos governos norte-americanos e europeus na criação de programas que incentivem o interesse feminino pelas carreiras em C\&T é um indicador do potencial econômico que este contingente feminino representa ${ }^{34,38}$. Um maior acesso feminino ao conhecimento científico e recursos tecnológicos é essencial para um melhor desempenho destes indivíduos em diferentes níveis da sociedade. Em países como o Brasil, onde a educação familiar assim como o ensino de primeiro e segundo graus são majoritariamente delegados às mulheres, os benefícios advindos da maior participação feminina em C\&T são explícitos ${ }^{39}$. Sob o aspecto econômico, a baixa proporção de mulheres em áreas científicas significa o desperdício de recursos humanos altamente qualificados que podem contribuir com soluções cientificamente criativas bem como diferentes pontos-de-vista. Em um contexto de globalização econômica e políticas nacionais de incentivo à crescente competitividade por novos mercados, este pode ser um fator limitante para o desenvolvimento científico e consequentemente para a produção de riquezas em uma nação visto que, desde a Revolução Industrial, descobertas científicas e tecnológicas historicamente vem sendo a mais importante ferramenta no acúmulo de bens pelos chamados países desenvolvidos. Sob o aspecto puramente científico, profissionais femininas contribuem para uma maior diversidade de abordagens e soluções para um dado problema. Uma maior representação feminina em $C \& T$ indubitavelmente enriquecerá o ambiente acadêmico através de novos talentos, valores e motivações. Afinal não é esta a época em que o estado-da-arte em ciência encontra-se na interface entre diferentes áreas? A receita de sucesso não é a criação de grupos formados por diferentes profissionais e, portanto, com diferentes visões e abordagens para um mesmo problema? Nada mais natural que aplicar este mesmo conceito às mulheres.

\section{ALGUMAS SOLUÇÕES ENCONTRADAS}

Certamente a presença de mulheres em ciência e tecnologia é mais alta nos Estados Unidos que nos demais países onde dados estatísticos são disponíveis. Um fator decisivo para isto foi a criação em 1981 do programa Women in Science com incentivo do $\mathrm{NSF}^{40}$. O programa levanta estatísticas a respeito do número de mulheres empregadas ou em treinamento em ciência e tecnologia, que regularmente é relatado ao Congresso e membros do governo americano. Como resultado desta primeira fase, foram implementados fundos para a premiação de jovens estudantes e pesquisadoras com bolsas de estudo e financiamento de projetos de pesquisa, bem como bolsas de curta duração para estudantes de segundo grau em laboratórios de pesquisas universitários, visando estimular o interesse de estudantes do sexo feminino por áreas de ciência e tecnologia. Um exemplo bem sucedido acontece no San Diego Super Computer Center (SDSC) onde há mais de três anos estudantes são recebidos com apoio do Programa e ampla participação de pesquisadores e professores ${ }^{40}$.

Um programa similar está sendo implementado nos países membros da Comunidade Européia chamado Women and Science $^{41}$. Nesta primeira etapa os objetivos do programa são dois: o desenvolvimento de diálogo entre os estados membros da Comunidade Européia e a criação de uma comissão que assegure a inclusão, no Fifth Framework, de mecanismos que estimulem o aumento da participação feminina em ciência. Efetivamente algumas medidas já estão em prática como a criação da bolsa Marie Curie para jovens estudantes e pesquisadoras visando não apenas estimular uma maior participação de mulheres em ciência e tecnologia, como o intercâmbio de conhecimento entre os estados membros. É significativa também a criação de uma rede de comunicação via internet por cada um destes programas ${ }^{40,42}$. Além de informações a respeito de bolsas de estudos/pesquisas e programas de pós-graduação, estes sítios possibilitam a troca de experiências entre jovens estudantes e pesquisadoras mais experientes, bem como a divulgação de encontros anuais que discutem a situação da mulher em Ciência e Tecnologia.

\section{CONCLUSÕES}

$\mathrm{O}$ crescente interesse, por parte dos governos federais e agências de financiamento, em incentivar a maior participação 
de mulheres em C\&T é uma expressão do importante potencial econômico que este grupo representa ${ }^{38,34}$. É impossível ignorar que este contingente feminino representa uma força de trabalho qualificada que vem sendo desperdiçada. Contudo, os aspectos sociais que podem advir de uma participação mais igualitária do sexo feminino em ciência não devem ser subestimados ${ }^{37}$. O acesso iqualitário à educação científica deverá contribuir significativamente para uma sociedade mais informada e qualificada para os desafios emergentes da revolução tecnológica e científica que o século passado presenciou ${ }^{39}$.

Com o recente debate iniciado pela Revista Nature ${ }^{3}$, espera-se que sociedades científicas se posicionem em relação à maior participação feminina em C\&T e, especialmente, que soluções sejam propostas. Uma série de medidas conjuntas entre governo e sociedades científicas vem sendo implementadas em diversos países. Nos EUA, a experiência do Massachussets Institute of Technology (MIT) tem servido como exemplo para outras instituições. A criação de programas de incentivo a estudantes e jovens cientistas, os quais premiam jovens estudantes e pesquisadoras com bolsas e financiamento de projetos de pesquisa, certamente tem sido um importante passo neste processo ${ }^{4}$. Como mulher e cientista penso ser fundamental a discussão do problema no Brasil. Muitos acreditam que o problema não existe, e certamente a mulher brasileira é bem menos discriminada do que as mulheres japonesas! No entanto, as sociedades norte-americanas e européias são mundialmente reconhecidas por uma política liberal e não discriminativa em relação à mulher e mesmo assim constatou-se que a discriminação sexual existe em instituições acadêmicas daqueles países. O Brasil não constitui uma exceção como muitos poderiam pensar pela ausência de discussão do tópico.

\section{AGRADECIMENTOS}

A autora agradece ao Prof. Ricardo de Carvalho Ferreira e aos revisores pelas valiosas correções e sugestões bibliográficas, e ao San Diego Super Computer Center (SDSC) pelo uso das facilidades computacionais e bancos de dados eletrônicos para a pesquisa bibliográfica.

\section{GLOSSÁRIO}

Machismo $^{43}$ - É um sistema de interrelações sociais entre indíviduos de diferentes sexos, o qual exagera diferenças entre homens e mulheres com basa nas chamadas "qualidades naturais" e determina que tipo de comportamento é aceitável por parte dos dois sexos. O machismo defende a superioridade masculina em relação à feminina, valorizando o comportamento viril, forte e agressivo em homens e estimulando um comportamento dependente, submissivo, emocional e de auto-sacrifício em mulheres. O machismo assim como o marianismo tem origens na cultura espânico-portuguesa colonial e nos ensinamentos da Igreja Católica.

Marianismo ${ }^{43}$ - Este comportamento social glorifica a maternidade como o principal papel social da mulher e defende a obrigação destas em atuar como guardiã dos valores morais da família, sempre dispostas ao auto-sacrifício na defesa da integridade familiar. O marianismo complementa perfeitamente o machismo, estimulando a divisão das funções sociais entre os dois sexos de modo que aos homens cabem as atividades públicas e de produção de riquezas e às mulheres cabem as atividades privadas e de procriação. As mulheres latino-americanas têm uma longa história de uso deste estereótipo cultural na obtenção de interesses sociais. Alguns movimentos de resistência na América Latina, como é o caso das Mães da Plaza de Mayo, foram bem sucedidos precisamente porque mulheres exploraram seu papel como mães. Este é um ícone poderoso, o qual governos e militares não quiseram ou puderam desafiar ${ }^{13,44}$.

\section{NOTA}

A busca bibliográfica para a elaboração deste artigo foi feita através do catálogo eletrônico da University of California e do San Diego Super Computer Center. Publicações nas seguintes áreas foram consultadas: 6.500 jornais na área de ciências; 1.300 jornais na área de psicologia; 1.500 magazines e jornais na área de ciências sociais; 43.000 citações em inglês, espanhol e português na área de estudos latino-americanos; 83.000 citações na área de ciências antropológicas; 700.000 citações na área de educação; 1.500 publicações governamentais, centenas de publicações em diferentes idiomas e na forma de livros, artigos, panfletos e bancos estatísticos na área de Public Affairs.

\section{REFERÊNCIAS}

1. Leslie, L. L. e Oxaca, R. L. In Higher Education: Handbook of Theory and Research Vol. 9; Eds.; Smart, J. C.; Agathon Press; New York, 1997; p 154.

2. Aisenberg, N. e Harrington, M.; Women of Academe: Outsiders in the Sacred Grove. University of Massachusetts Press; Amherst, MA, 1988; 207 p.

3. Durante a submissão deste artigo, a revista Nature discutiu a questão da discriminação sexual em Ciência e Tecnologia com ampla participação da comunidade científica. O debate é aberto ao público, e uma extensa referência bibliográfica sobre o assunto pode ser encontrado no web site da revista, e o acesso é gratuito à toda a comunidade. O endereço eletrônico é http://imagine.nature.com/debates/ women/women_frameset.html

4. Women, Minorities and Persons with Disabilities in Science and Engineering: 1998. NFS, Arlington, USA, 1999. http://www.nsf.gov/sbe/srs/nsf99338/frames.htm

5. Wennerås, C. ; Wold, A.; Nature 1997, 387, 341.

6. Grant, J ; Burden, S; Breen, G; Nature 1997, 390, 438.

7. Lane, N; Nature 1999, 401. http://imagine.nature.com/debates/women/

8. Pardue, M-L; Hopkins, N.; Potter, M. C.; Ceyer, S.; Nature 1999, 401. http://imagine.nature.com/debates/women/

9. The MIT Faculty Newsletter, special edition 1999, XI, 4. http://web.mit.edu/fnl/women/women.html

10. Peltonen, L. In Women in Science: Mobilizing to Enrich European Research; Eds.; Colosimo, A. e Dewandre, N.; Luxembourg; 1999; pp 65-68. http://www.cordis.lu/ improving/src/women_conf.htm

11. Krummacher, S.; Nature 1999, 401. http://imagine.nature.com/ debates/women/

12. Dewandre, N.; Nature 1999, 401. http://imagine.nature.com/ debates/women/

13. Twombly, S.; Higher Education 1998, 35, 367.

14. Kanter, R. M. Men and Women of the Corporation. Basic Books, Inc., New York, 1993; 390 p.

15. Epstein, C. F.; Deceptive distinctions : sex, gender, and the social order; New Haven, Yale University Press, 1988.

16. Friedman, L.; Review of Educational Research 1989, 59, 185.

17. Orenstein, P.; School girls : Young Women, Self-esteem, and the Confidence gap. Anchor Books, New York, 1995.

18. Linn, M. C. e Hyde, J. S.; Educational Research 1989a, $18,17$.

19. Linn, M. C. e Hyde, J. S.; Educational Research 1989b, $18,22$.

20. Hyde, J. S.; Fennema, E. e Lamon, S. J.; Psychological Bulletim 1990, 107, 139.

21. Meece, J. L.; Parson, J. E.: Kaczala, C. M.; Goff, S. R. e Futterman, R. Psychological Bulletin 1982, 91, 324.

22. Leslie, L. L.; McClure, G. T. e Oxaca, R. L.; The Journal of Higher Education 1998, 69, 239.

23. Astin, A. W.; What matters in College? Four Critical Years Revisited. Jossey-Bass, San Francisco, 1993. 
24. Lent, R. W.; Lopez, F. G. e Bieschke, K. J.; Journal of Counseling Psychology 1991, 38, 424.

25. Betz, N. E. e Hackett, G.; Journal of Vocational Behavior 1983, 23, 329

26. Wainer, H. e Steinberg, L. S.; Harvard Educational Review 1992, 62, 323.

27. Marsh, H. W.; American Educational Research Journal 1989, 26, 191

28. Rudisill, E. M. e Morrison, L. J.; School Science and Mathematics 1989, 89, 571.

29. Benbow, C. P. e Stanley, J. C.; Science 1983, 222, 1029.

30. McNeil, L.; Sher, M.; Report on the dual-career-couple survey. http://www.physics.wm.edu/ sher/survey.html

31. Stevenson, S.; Nature, 1997, 390, 201.

32. Ebeling, H; Nature 1999, 402.

33. Maisel, M.; Smart, L.; Women in Science: A Selection of 16 Significant contributors. San Diego Supercomputer Center, San Diego, CA, 1997; 100 p.

34. HESA Statistics in The Rising Tide: A report on Women in Science, Engineering and Technology; HMSO, London, 1994.

35. Abbot, A; Nature 1998, 393, 402.

36. Gavaghan, H; Nature 1997, 390, 230.

37. Women, Science and Technology in Latin America:
Diagnosis and Strategies. Bariloche, Argentine, 1998. http://helix.nature.com/wcs/m20s.html

38. Realising our Potential - A Strategy for Science, Engineering and Technology; HMSO, London, 1993.

39. Third World Organization for Women in Science. Conference Statement of the Second General Assembly and International Conference: Women, Science and Technology for Sustainable Human Development. Cape Town, South Africa, 1999. http://helix.nature.com/wcs/m02s.html

40. Association for Women in Science. http://www.awis.org/

41. San Diego SuperComputer Center Scholar Program for Young Women. http://www.sdsc.edu/Scholars/scholars.html

42. The European Commission for Women and Science. http://www.cordis.lu/improving/src/hp_women.htm

43. Fisher, J.; Out of the shadows: women, resistance and politics in South America. Latin America Bureau, London, 1993.

44. Winn, P.; Americas: The changing face of Latin America and the Caribbean. The University of California Press, Berkeley, 1992.

45. Azevedo, E. S.; Fortuna, C. M. M.; Pontes, M. C. D., Freire, N. B. V. M. e Dórea, E. S. Ciencia e Cultura, 1989, 4, 275.

46. Todos os números mencionados nesta citação foram obtidos no web site do CNPq. http://www.cnpq.br. 